Original Research Paper

\title{
Survey on Early Detection of Alzheimer's Disease using Different Types of Neural Network Architecture
}

\section{Deepthi Kamath $^{1}$, Misba Firdose Fathima ${ }^{1}$, Monica K. P ${ }^{1}$, M Kusuma ${ }^{1}$}

${ }^{1}$ Department of Information Science and Engineering. Dayananda Sagar Academy of Technology and Management, Bangalore. Karnataka, India.

Article History

Received:

23.03.2021

Revised:

09.06.2021

Accepted:

12.06.2021

*Corresponding Author:

Deepthi Kamath

Email:

deepthikamath297@gmail.com

This is an open access article, licensed under: $\mathrm{CC}-\mathrm{BY}-\mathrm{SA}$

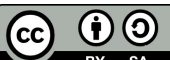

Abstract: Alzheimer's disease is a condition that leads to, progressive neurological brain disorder and destroys cells of the brain thereby causing an individual to lose their ability to continue daily activities and also hampers their mentality. Diagnostic symptoms are experienced by patients usually at later stages after irreversible neural damage occurs. Detection of $\mathrm{AD}$ is challenging because sometimes the signs that distinguish AD MRI data, can be found in MRI data of normal healthy brains of older people. Even though this disease is not completely curable, earlier detection can aid in promising treatment and prevent permanent damage to brain tissues. Age and genetics are the greatest risk factors for this disease. This paper presents the latest reports on $\mathrm{AD}$ detection based on different types of Neural Network Architectures.

Keywords: Alzheimer's Disease, Convolutional Neural Network, Magnetic Resonance Imaging. 


\section{Introduction}

Alzheimer's disease is a condition that affects the brain. Though the symptoms are mild at first it becomes more severe over time. Common symptoms of AD include memory loss, language problems, and impulsive or unpredictable behavior. As the symptoms get worse, it becomes hard for people to recollect recent events and they also find it difficult to recognize familiar faces. AD can range from a state of mild impairment, to moderate impairment, before eventually reaching severe cognitive decline [1].

People with Mild AD develop memory problems as well as cognitive difficulties that may take longer than usual to perform daily tasks, wandering and getting lost. In Moderate AD, damages are done to the parts of the brain which are responsible for language, senses, reasoning, and consciousness. In Severe AD, plaques and tangles are present throughout the brain, causing the brain tissue to shrink substantially [1].
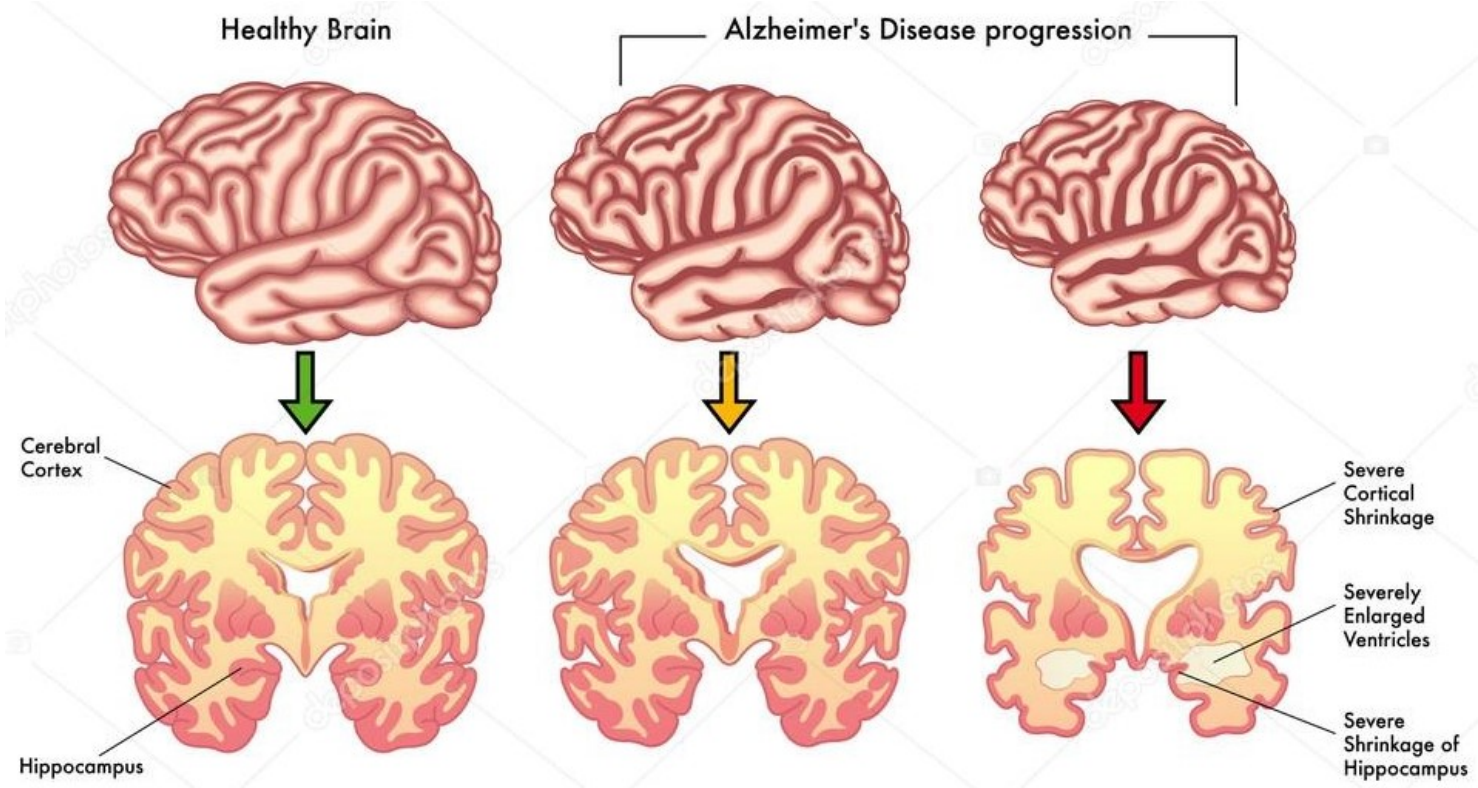

Figure 1. Symptoms of Alzheimer's Disease [2]

Hippocampus is responsible for the episodic and spatial memory of the brain. The reduction in the hippocampus leads to cell loss and specifically affects synapses and neuron ends thus neurons cannot communicate anymore via synapses [2]. As an outcome, brain regions offering remembrance (short term memory), thinking ability, planning, and judgment are affected. In elderly individuals over the age of 75, identifying differences between AD brain and a normal functioning brain is difficult as they share similar brain patterns and image intensities [1].

As per the reports, the below pie chart gives us a clear picture of the people who are mostly affected by $\mathrm{AD}$ considering age as an element.

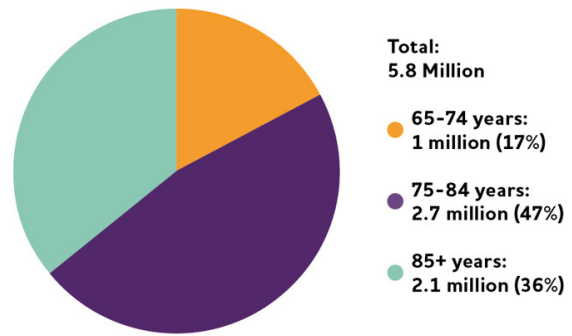

Figure 2. Pie-chart of Patients affected by Alzheimer's Disease Considering AGE as a Factor [3] 


\section{Types of Neural Network}

Artificial Neural Network techniques are used for prediction and classification. These concepts have been utilized in various fields, that includes natural language processing and computer vision, both of which demonstrate significant increase in the performance. Hybrid approaches have often lent us with good results, but they rarely take advantage of neural networks, which automatically highlights and extracts features from large sets of neuroimaging data [4].

\subsection{Artificial Neural Network}

Artificial Neural Network (ANN) is a collection of multiple neurons in each and every layer. ANN is sometimes referred to as Feed-Forward Neural network as the data is always processed in the forward direction. ANN consists of 3 layers which are Input, Hidden and Output layers [5]. The input layer fetches all the inputs, on the other hand hidden layer processes and analyses the inputs that were fetched previously, and at the end the output layer produces the result for the same. It is observed that each layer tries to learn certain weights.

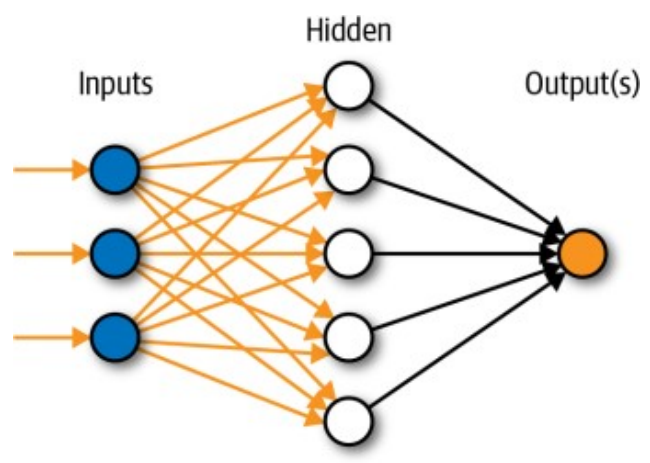

Figure 3. Artificial Neural Network [6]

Advantages of ANN [5]:

1. ANN has the ability to learn about nonlinear functions. Therefore, the networks are known as Universal Function Approximators. ANN can learn about the weights by mapping the inputs and the outputs.

2. Major reason behind universal approximation is the activation function. Activation function represents nonlinear functionalities to the network. This allows the network to learn many complex relationships that exist between the input and the output.

\subsection{Recurrent Neural Networks}

Recurrent Neural Networks (RNN) is a unique type of network, which unlike feedforward networks has recurrent connections. RNN initially holds a recurrent connection in the hidden state. This looping constraint ensures that sequential information is obtained from the input data. Therefore, when a looping constraint is applied on the layer of ANN it automatically shifts to RNN [6].

Advantages of RNN [5]:

1. RNN acquires the information which is sequentially present in the input data i.e. making predictions of the dependency existing between the words in a given text.

2. RNNs provide different parameters across various time steps. This is popularly known as Parameter Sharing. This results in fewer parameters to train and decreases the computational cost. 


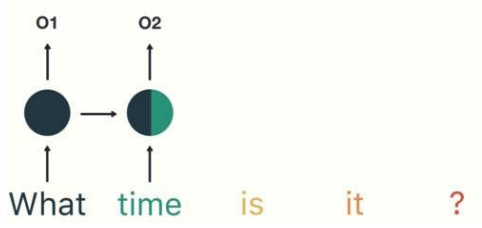

Figure 4. Many-2-Many Seq-2-Seq model [7]

Figure 5 shows the output (o1, o2, o3, o4) at each time step depends not only on the current word but also on the previous words.

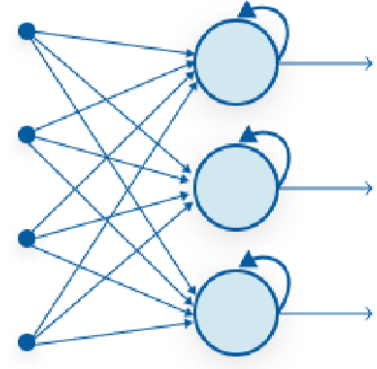

Recurrent Neural Network

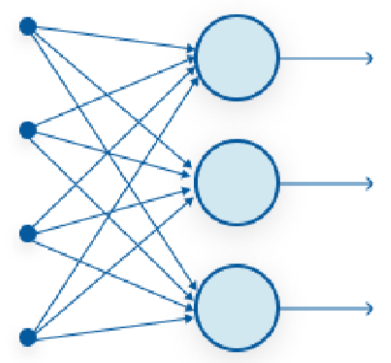

Feed-Forward Neural Network

Figure 5. Recurrent Neural Network [5]

\subsection{Convolutional Neural Network}

Convolutional Neural Network (CNN) is a deep Feed-forward Neural Network (FNN) that comprises multilayers of artificial neurons, which provides good performance in large-scale segmentation, processing of image, classification and also for other auto correlated data. The foundation for CNNs are filters i.e. kernels. Kernels use the convolution operation in order to fetch all the relevant features from the input. The importance of filters using images as input data is as shown in the figure below.

$\mathrm{CNN}$ was initially introduced to solve problems related to image data. But it has been observed that they perform significantly well on sequential inputs as well [5].

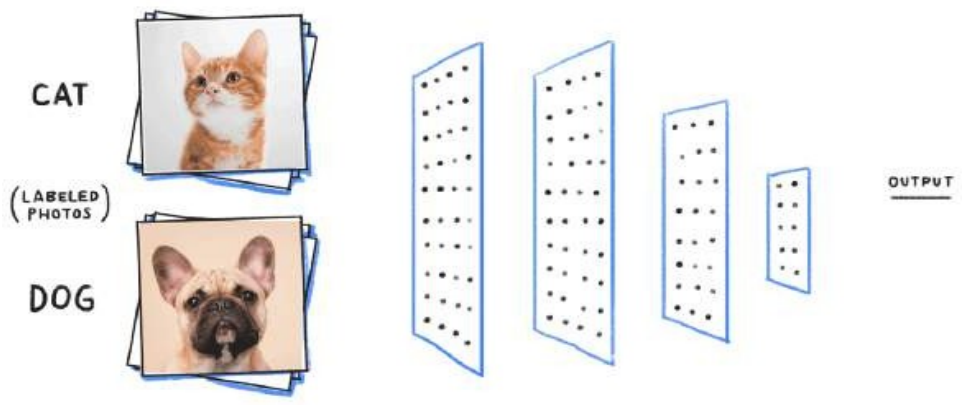

Figure 6. Convolutional Neural Network - Image Classification [8] 
Advantages of $\mathrm{CNN}$ :

1. CNN automatically learns about the filters without referring to it explicitly. They come in handy during the extraction of relevant features from the input data.

2. CNN fetches the spatial features of an image. Spatial features refer to the pixel arrangement and the relationship between the pixels in an image. They aid us in determining the object accurately, the location of an object, as well as its relationship existing between other objects in an image.

\subsection{Deep Learning}

Deep learning (also known as deep structured learning) comes under one of the main branches of machine learning methods which is based on artificial neural networks that provides the ability for computers to learn from previous experience and understand the world in terms of hierarchy of concepts. The computer analyses data and gathers knowledge from experience, thereby reducing the need for a physical operator formally to specify all of the knowledge needed by the computer.

The vast distribution of concepts allows the computer to comprehend complicated concepts by building them out of simpler ones [9] [10].

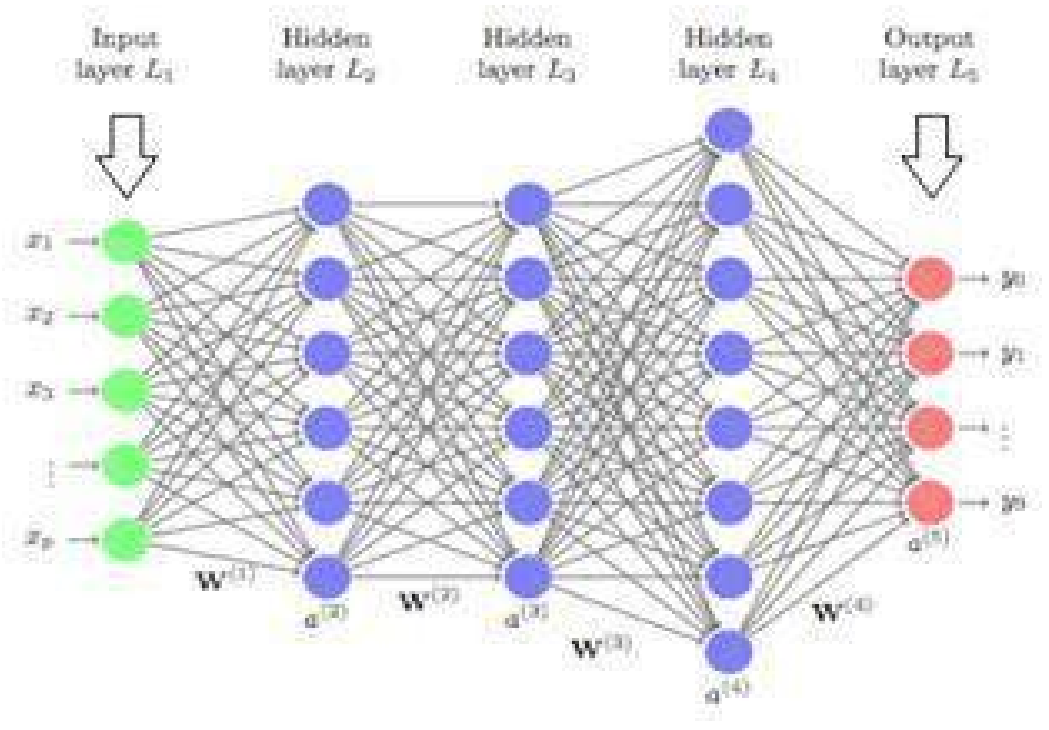

Figure 7. Simple Artificial Neural Network with Three Hidden Layers [11]

Deep learning architectures such as deep neural networks, deep belief networks etc. have been integrated to various fields which includes speech recognition, natural language processing, computer vision, machine vision, medical image analysis, material inspection and board game programs where the results obtained are not only comparable but at the same time surpasses human expert performance [9].

\section{Existing Methodologies}

Various methods of the Neural Network have been implemented in the state-of-the-art literature. NN's ability to adapt according to varying input, allows the network to generate best possible results without needing to modify the output criteria.

Here, Islam, et al. [1] has deployed three Dense Net styled models - DenseNet-121, DenseNet-161, and DenseNet-169 accordingly. Every MRI data has patches that are created from three planes of imaging: Coronal or frontal plane, Sagittal or median plane and Axial or horizontal plane. These patches are given as an input to the proposed network. They've used the following: transfer learning, three models (pre-trained) with Image Net dataset. Each model is optimized with the Stochastic Gradient Descent (SGD) algorithm to achieve $83.18 \%$ overall accuracy. 
The back-propagation algorithm is used by Taeho et al. [12] to determine the error present between the network output and the expected output in Gradient Computation. Once the initial error value is determined by using least squares method, the weights are updated until the differential value becomes 0.To improve this performance, multimodal neuroimaging data such as amyloid PET, FDGPET and MRI have been used for determining brain amyloid- $\beta$ accumulation, glucose metabolism and brain structural atrophy respectively. Deep learning approaches have to $86.0 \%$ accuracy for AD classification and $84.2 \%$ for MCI conversion prediction.

The architecture is built using Keras with TensorFlow backend by Yagis, et al [13]. In Data preprocessing: here the data are modified into a standardized structure. This is achieved by performing co-registration with a skull stripping and standard template.

A 3D CNN model is built after getting inspired by VGG-16 architecture. The model has obtained training with Adam optimizer and categorical cross-entropy loss. 3D models are used here to avoid information loss. The average accuracy of the model achieved is $73.4 \%$ for the dataset (ADNI) and $69.9 \%$ classification accuracy for the OASIS dataset.

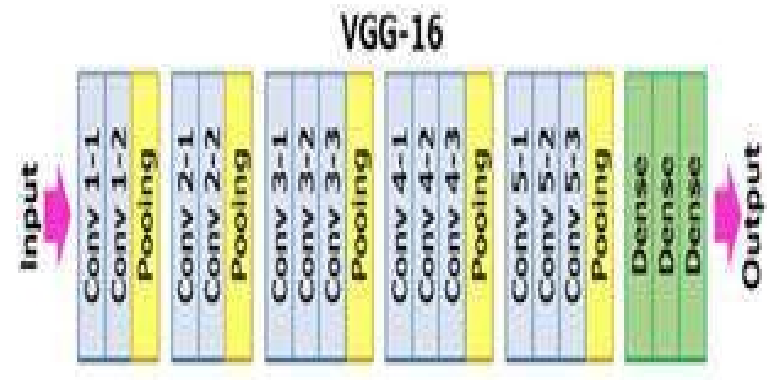

Figure 8. 3D VGG-16 Architecture [12]

First the "Data Type Analysis" is done by Huang, et al. [14], where the determination of proper types of data and ROIs are obtained. In order to know the effect of segmentation, they have segmented the cognitively unimpaired subjects and AD of T1-MRI with the assistance of MALP-EM algorithm and therefore obtained the datasets that are segmented. Later, a set of VGG-like MultiModality AD classifiers is built, which considers both FDG-PET and T1-MRI data as input parameters and provides predictions accordingly. Then they tested the networks with the pMCI and sMCI data. MCI is further classified into progressive MCI (pMCI) and stable MCI (sMCI). Distinguishing between pMCI and sMCI plays a huge role in the early diagnosis and treatment of dementia.This network is programmed based on TensorFlow. Training procedures of the networks are conducted on a personal computer with a GPU.

The first step by Mehmood, et al. [15] was data augmentation and preprocessing the second stage involved extraction of features from input images, and the third step was the division of dementia classes. They have come up with a CNN - based approach idealized from VGG-16 for the classification and determination of dementia stages.

Saman, et al. [16], have used a very deep CNN structure in order to adopt binary classification. The scale and shift invariant features are fetched from various layers of $\mathrm{CNN}$ architecture resulting in a very accurate well trained model. Later, unique preprocessing strategies utilized in this work brought about significant enhancement in the quality of the data fed into LeNet and GoogleNet which impacted positively towards the classifier performance.

Al-Azdi et al [17], marked the datasets where $75 \%$ is used for training $25 \%$ is used for data testing purposes and also for binary classification. This data collection was preprocessed before by undergoing training and testing. The structure of neural networks is using Alexnet architecture with ve layer of convolution. Compared to other journal results, the study suggests uses of LeNet or GoogleNet architecture and ADNI database.

Scans from the MRI are delivered as 3D Nifti volumes. At first, grey matter (GM) segmentation and skull stripping is carried out on an axial scans through spatial normalization bias correction along with modulation using SPM-g* component by Farooq et al. [18]. GM volumes are then converted to 
JPEG slices using the Python Nibabel package. Slices on the either ends with no information are omitted from the data set.

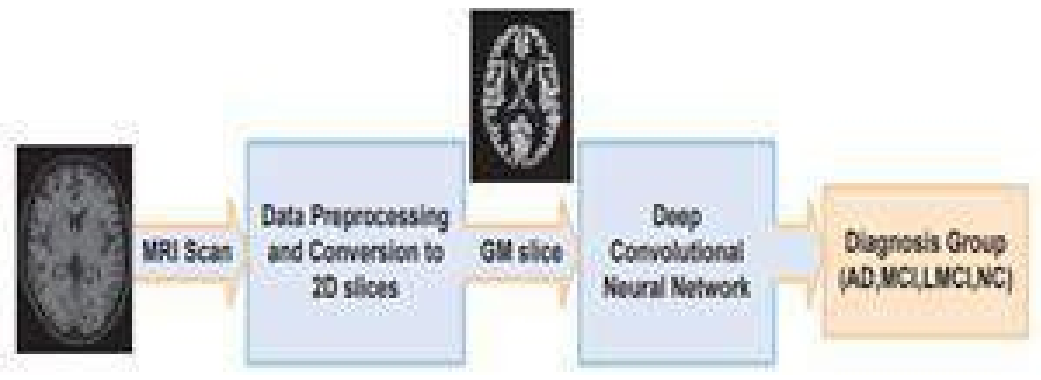

Figure 9. Deep Learning Pipeline [8]

Table 1. Comparison of the Method, Datasets and Accurancy

\begin{tabular}{|c|c|c|c|}
\hline Paper & Method & Datasets & Accuracy (\%) \\
\hline$[10]$ & DenseNet & T1-MRI & 83.18 \\
\hline$[12]$ & 3D CNN & T1-MRI, FDG-PET & 91.09 \\
\hline$[13]$ & VGG-16 & $\begin{array}{c}\text { T1-MRI } \\
\text { ADNI-73.4 OASIS- }\end{array}$ & 69.9 \\
\hline$[14]$ & VGG based on & MRI, PET CN/AD-77.2 & 65.28 \\
& ALEXNET & CN/pMCI-70 sMCI/pMCI- & \\
\hline$[15]$ & VGG-16 & MRI & $89.05 \%$ \\
\hline$[17]$ & LeNet GoogLeNet & MRI & $87.4 \%$ \\
\hline$[18]$ & GoogLeNet ResNet- & T3-MRI & $88.88 \% 5$ \\
& 18 ResNet-152 & & $88.41 \%$ \\
\hline$[19]$ & GoogLeNet & rs-fMRI & $88.84 \%$ \\
\hline
\end{tabular}

\section{Conclusion}

From this survey, we can draw a conclusion that there are various technologies and methodologies used for detection of Alzheimer's disease at an earlier stage where each individual methodology has a variable precision and accuracy. The two primary datasets, namely ADNI and OASIS are being used commonly. CNN based Classification model is used to predict Alzheimer's disease affected-brain v/s a normal aging brain and is able to do so with higher accuracy.

Therefore we can conclude that CNN method can be used as it has the highest accuracy than the other methods which were used.

\section{References}

[1] Anonymous, "Stages of Alzheimer's", Alzheimer's Association", 2021. [Online]. Available: https://www.alz.org/alzheimers-dementia/stages. [Accessed: January, 2021]. 
[2] Anonymous, "Alzheimer's Medical Illustration of the Symptoms", 2021. [Online]. Available: https://st2.depositphotos.com/2852841/12083/v/950/depositphotos_120837382-stock-illustrati on-medical-illustration-of-the-symptoms.jpg. [Accessed: January, 2021].

[3] I. Jyoti, and Y. Zhang, "Ensemble of Deep Convolutional Neural Networks for Alzheimer's Disease Detection and Classification," arXiv preprint arXiv:1712.01675, 2017.

[4] Report 2020, "2020 Alzheimer's Disease Facts and Figures", the Journal of Alzheimer's Association. https://doi.org/10.1002/alz.12068, 2020.

[5] A. Pai, "CNN vs. RNN vs. ANN: an Analyzing 3 Types of Neural Networks in Deep Learning, February", 17, 2020. [Online]. Available: https://www.analyticsvidhya.com/blog/2020/02/cnnvs-rnn-vs-mlp-analyzing-3-types-of-neural-networks-in-deep-learning/ [Accessed: January, 2021].

[6] Anonymous, “Artificial Neural Network", February, 2021. [Online]. Available: https://images.squarespace-cdn.com/content/v1/5ccb715016b640627a1c2782/ [Accessed: January, 2021].

[7] Anonymous, "Analyticsvidhya", 2021. [Online]. Available: https://cdn.analyticsvidhya.com/ wp-content/uploads/2020/02/1d_POV7c8fzHbKuTgJzCxtA.gif [Accessed: January, 2021].

[8] Anonymous, "Analyticsvidhya", 2021. [Online]. Available: https://cdn.analyticsvidhya.com/ wp-content/uploads/2020/02/1oB3S5yHHhvougJkPXuc8og.gif [Accessed: January, 2021].

[9] Anonymous, "Deep Learning, 2021. [Online]. Available: https://en.wikipedia.org/wiki/Deep_ learning [Accessed: January, 2021].

[10] I. Goodfellow, Y. Bengio, A. Courville, and Y. Bengio, Deep learning. Cambridge: MIT Press, 2016.

[11] Anonymous, "Deep Learn", 2021. [Online]. Available: https://orbograph.com/wpcontent/uploads/2019/01/DeepLearn.png [Accessed: January, 2021].

[12] J. Taeho, K. Nho, and A. J. Saykin, "Deep learning in Alzheimer's disease: diagnostic classification and prognostic prediction using neuroimaging data", Frontiers in Aging Neuroscience, vol. 11, pp. 220, 2019.

[13] E. Yagis, L. Citi, S. Diciotti, C. Merzi, W. S. Atnafu, and G. S De Herrera, “ 3D Convolutional Neural Networks for Diagnosis of Alzheimer's Disease via structural MRI”, 2020.

[14] Y. Huang, J. Xu, Y. Zhou, T. Tong, and X. Zhuang, "Alzheimer's Disease Neuroimaging Initiative (ADNI)", Diagnosis of Alzheimer's disease via multi-modality $3 D$ convolutional neural network. Frontiers in Neuroscience, vol. 13, pp. 509, 2019.

[15] A. Mehmood, M. Maqsood, M. Bashir, and Y. Shuyuan, "A Deep Siamese Convolution Neural Network for Multi-Class Classification of Alzheimer Disease. Brain Sciences", vol. 10, no. 2, pp. 84, 2020.

[16] S. Saman, and G. Tofighi, "Classification of alzheimer's disease structural MRI data by deep learning convolutional neural networks", arXiv preprint arXiv:1607.06583,2016.

[17] F. Al-Azdi, R. Passarella, A. Susanto, C. Caroline, R. D. Puspa and T. W. Yudha, "Design of A Convolutional Neural Network System to Increase Diagnostic Efficiency of Alzheimer's Disease", in IOP Conference Series: Materials Science and Engineering, IOP Publishing, vol. 648, no. 1, October, 2019.

[18] A. Farooq, S. Anwar, M. Awais, and S. Rehman, "A deep CNN based multi-class classification of Alzheimer's disease using MRI ", in IEEE International Conference on Imaging systems and Techniques (IST) IEEE, pp. 1-6, October 2017.

[19] S. Saman, and G. Tofighi, "Alzheimer's Disease Neuroimaging Initiative", DeepAD: Alzheimer's disease classification via dkr7eeep convolutional neural networks using MRI and fMRI. BioRxiv, pp. 070441, 2016. 